\title{
Laparoscopic evaluation and prevalence of endometriosis among infertile women: a prospective study
}

\author{
Sana Sharfuddin', Nazar Imam², Anup Pradhan ${ }^{1 *}$
}

\begin{abstract}
${ }^{1}$ Department of Obstetrics and Gynecology, Sikkim Manipal Institute of Medical Sciences Gangtok, Sikkim, India ${ }^{2}$ Department of Surgery, Sikkim Manipal Institute of Medical Sciences Gangtok, Sikkim, India
\end{abstract}

Received: 15 December 2019

Revised: 19 February 2020

Accepted: 19 March 2020

\author{
*Correspondence: \\ Dr. Anup Pradhan, \\ E-mail: anuppradhan@live.com
}

Copyright: () the author(s), publisher and licensee Medip Academy. This is an open-access article distributed under the terms of the Creative Commons Attribution Non-Commercial License, which permits unrestricted non-commercial use, distribution, and reproduction in any medium, provided the original work is properly cited.

\begin{abstract}
Background: Endometriosis can be defined as presence of endometrial mucosa outside the uterine cavity. There are no specific signs and symptoms of endometriosis and the presentation may vary depending upon the site of involvement. It is one of the common causes of subfertility and infertility in young women. The Management of endometriosis may include medical as well as surgical intervention. Laparoscopic interventions have found to have encouraging results in terms of increasing chances of fertility in young women with endometriosis.

Methods: This was a prospective study in which women who had infertility and subsequently diagnosed to be having endometriosis were included. A detailed history was taken with particular emphasis on duration of symptoms, presence of additional co-morbidities and duration of infertility was recorded. Diagnostic laparoscopy was done in all the cases. Depending upon location and severity of endometriosis adhesiolysis, excision and ablation of endometrial lesions was done. Patients were followed up and successful pregnancies (spontaneous as well as secondary to assisted reproductive techniques) were recorded.

Results: Out of 200 studied cases endometriosis was seen in $23(11.5 \%)$ patients. the most common affected age group was between $26-30$ years $(47.83 \%)$. 18 patients $(78.26 \%)$ had primary infertility whereas remaining 5 patients $(21.74 \%)$ were found to have secondary infertility. The most common site was found to be ovaries which was found to be involved in all the cases. cystectomy (30.43\%), endometrioma drainage and fulguration (13.04\%) or adhesiolysis (34.78\%) were the commonly done surgical interventions in cases. out of 18 patients who had successful ovulation following laparoscopic intervention 12 (66.66\%) patients completed their pregnancies successfully.

Conclusions: Laparoscopic interventions in patients with infertility have diagnostic as well as therapeutic value and are found to have good outcome in terms of successful pregnancy.
\end{abstract}

Keywords: Endometriosis, Infertility, Laparoscopic interventions, Successful pregnancy

\section{INTRODUCTION}

Endometriosis can be defined as presence of endometrial mucosa outside the uterine cavity. There are no specific signs and symptoms of endometriosis and the presentation may vary depending upon the site of involvement. ${ }^{1}$ It may manifest as dysmenorrhea, irregular menstrual bleeding, nonspecific low back pain, dyspareunia, abdominal pain and urinary complaints. One of the important features of women with endometriosis is infertility and more than $25 \%$ of the patients have either infertility or subfertility. The common locations where ectopic endometrial mucaosa is found include but is not limited up to ovaries, cervix, vagina, fallopian tubes and uterine ligaments. ${ }^{2}$ The uncommon locations for endometriosis include visceral organs (spleen, lungs and 
kidneys), nasal mucosa, stomach and pleura. Endometriosis at these unusual places may present as bizarre but very characteristic signs and symptoms such as cyclic epistaxis or cyclic haemoptysis occurring at the time of menstrual cycles. ${ }^{3}$

The exact etiology of endometriosis still remains an enigma for investigators. Various theories have been proposed but none have been conclusively proven. The commonly believed theories include metaplastic conversion of epithelium and hematogenous or lymphatic spread of endometrial epithelial cells from within endometrial cavity to ectopic locations. Irrespective of the exact etiology the risk factors for endometriosis include presence of a family member with endometriosis, early menarche, low parity and nulliparity. ${ }^{4}$ Though it is reported that approximately $5-10 \%$ of women suffer from endometriosis it's difficult to estimate exact prevalence of endometriosis because its definitive diagnosis depends upon demonstration of endometrium at ectopic sites by invasive procedures such as biopsy, laparoscopy or laparotomy. ${ }^{5}$

There are many ways in which endometriosis adversely affect the fertility of women. Various mechanisms responsible for subfertility in these cases include impaired oocyte release due to endometriosis. Tubal blockage and impaired tubal transport owing to tubal endometriosis and failure of sperm migration. The management of patients who has been diagnosed with infertility and endometriosis may consist of medical or surgical management and in many cases a combination of medical and surgical management may be required. ${ }^{6}$

Imaging studies an important role in first suspecting the diagnosis of endometriosis. Ultrasonography is routinely done in these patients and it may show presence of homogenous, focal lesions with low-level echoes devoid of vascularity on doppler scanning. In some cases imaging studies such as magnetic resonance imaging can be helpful in determining the extent of the disease. ${ }^{7}$ Despite all these imaging studies in many women with infertility laparoscopy is recommended. Though invasive it is considered as gold standard for the definitive diagnosis and management of infertility associated with endometriosis. It may further help in management of endometriosis since macroscopic endometriosis can be removed by surgical intervention. More recently the trend is shifting towards laparoscopic evaluation of these patients and laparoscopic interventions are being preferred over laparotomy because of the obvious advantage of minimal damage to the healthy tissue, quick recovery, early mobilization and comparatively less hospital stay. Laparoscopic interventions moreover have a unique advantage of magnification which is crucial in cases in whom there are small foci of endometriosis. ${ }^{8}$

Study conducted this prospective study of laparoscopic evaluation of patients of endometriosis having infertility.

\section{METHODS}

This was a prospective cohort study which was conducted in the department of obstetrics and gynecology of a tertiary care medical college situated in an urban area. All the patients who had infertility and subsequently diagnosed to be having endometriosis were included in this study on the basis of a predefined inclusion and exclusion criteria.

Demographic details such as age, weight and socioeconomic status was recorded. A detailed history was taken with particular emphasis on duration of symptoms, presence of additional co-morbidities and duration of infertility was recorded. A detailed clinical examination was done in all the cases. All patients underwent basic investigations such as complete blood count, renal and hepatic functions, random blood sugar levels and bleeding and clotting time. Ultrasonography was done in all the cases. Diagnostic laparoscopy was done in all the cases. Depending upon location and severity of endometriosis adhesiolysis, excision and ablation of endometrial lesions was done. Post laparoscopy patients were assessed clinically as well as by ultrasonography. Patients were followed up and successful pregnancies (spontaneous as well as secondary to assisted reproductive techniques) were recorded. Cystectomy and fulguration were done in appropriate cases.

Endometriosis staging was done using the RevisedAmerican Fertility Society (R-AFS) classification score. Scores 1 to 5 were classified as endometriosis stage 1 (minimal), whereas scores from 6-15 were labelled as stage 2 endometriosis (mild), scores 16-40 with minimal adhesions were classified as stage 3 endometriosis (moderate) and scores above 40 were classified as stage 4 (severe).

\section{Inclusion criteria}

- Patients having infertility (inability to conceive despite 12 months of unprotected intercourse)

- $\quad$ Age between 18-40 years

- Endometriosis was confirmed by laparoscopy

- Patients who gave informed written consent to be part of the study.

\section{Exclusion criteria}

- Those who refused consent

- Presence of associated male factors of infertility

- Patients with severe co-morbid conditions

- $\quad$ Age more than 40 years.

\section{Statistical analysis}

The statistical analysis was done using SSPS 21.0 software. 


\section{RESULTS}

A total of 200 patients with infertility were included in this study on the basis of a predefined inclusion and exclusion criteria. Out of 200 patient's endometriosis was seen in $23(11.5 \%)$ patients (Figure 1).

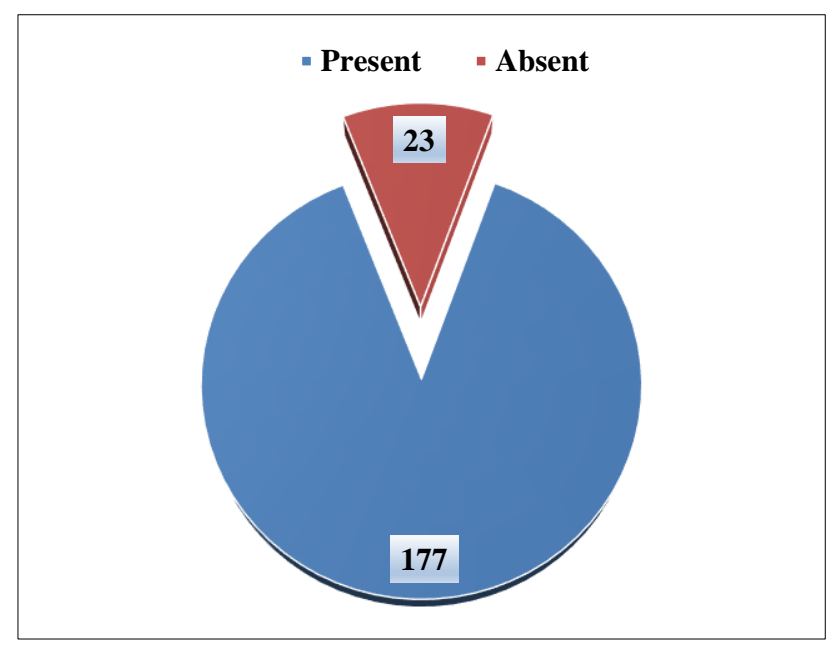

Figure 1: Endometriosis in studied cases.

Table 1: Age distribution of the studied cases.

\begin{tabular}{|lll|}
\hline Age & No. of patients & Percentage \\
\hline 18-25 years & 3 & $13.04 \%$ \\
\hline 26-30 years & 11 & $47.83 \%$ \\
\hline 31-35 years & 7 & $30.43 \%$ \\
\hline 36-40 years & 2 & $8.70 \%$ \\
\hline Total & 23 & $100 \%$ \\
\hline
\end{tabular}

The analysis of the patients on the basis of age showed that the most common affected age group was between 26-30 years $(47.83 \%)$ followed by $31-35$ years $(30.43 \%)$. Comparatively less number of patients were seen below 25 years $(13.04 \%)$ and above 35 years $(8.70 \%)$ of age (Table 1).

Table 2: Socio-economic status of the studied cases.

\begin{tabular}{|lll|}
\hline Socio economic status & Patients with endometriosis \\
\hline & No. of cases & Percentage \\
\hline Upper class & 0 & $0.00 \%$ \\
\hline Upper middle class & 1 & $4.35 \%$ \\
\hline Middle class & 3 & $13.04 \%$ \\
\hline Lower middle class & 7 & $30.43 \%$ \\
\hline Lower class & 12 & $52.17 \%$ \\
\hline Total & 23 & $100 \%$ \\
\hline
\end{tabular}

The distribution of the patients on the basis of modified Kuppuswamy scale showed majority of the patients $(52.17 \%)$ belonged to lower class followed by lower middle class $(30.43 \%)$ and middle class $(13.04 \%)$ (Table 2).
Out of 23 patients who had been found to have endometriosis 18 patients $(78.26 \%)$ had primary infertility whereas remaining 5 patients $(21.74 \%)$ were found to have secondary infertility (Table 3 ).

Table 3: Type of infertility in studied cases.

\begin{tabular}{|lll|}
\hline Type of infertility & No. of patients & Percentage \\
\hline Primary & 18 & $78.26 \%$ \\
\hline Secondary & 5 & $21.74 \%$ \\
\hline Total & 23 & $100 \%$ \\
\hline
\end{tabular}

The analysis of patients having endometriosis showed that out of 23 patients 11 patients had co-morbid conditions. The most common associated condition was found to be polycystic ovaries which was seen in 6 patients $(26.09 \%)$, hypertension was present in 3 patients $(13.04 \%)$. Diabetes and uterine fibroid was present in 1 patient each $(4.35 \%)$ (Figure 2).

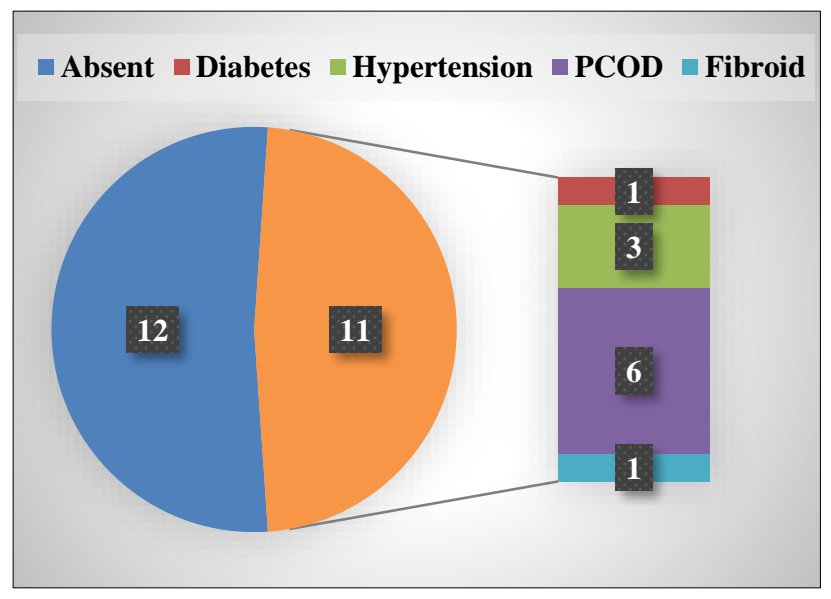

Figure 2: Co-morbid conditions in studied cases.

Table 4: Sites of endometriosis seen during laparoscopy.

\begin{tabular}{|llll|}
\hline \multicolumn{2}{|l}{ Socio economic status } & \multicolumn{2}{l|}{ Patients with endometriosis } \\
\hline \multirow{3}{*}{ Ovaries } & No. of cases & Percentage \\
\cline { 2 - 4 } & Right & 5 & $21.74 \%$ \\
\cline { 2 - 4 } & Left & 7 & $30.43 \%$ \\
\hline Uterine walls & Both & 11 & $47.83 \%$ \\
\hline Uterine ligaments & 3 & $13.04 \%$ \\
\hline POD & 2 & $8.70 \%$ \\
\hline
\end{tabular}

The analysis of site of endometriosis showed that the most common site was found to be ovaries which was found to be involved in all the cases. Endometriosis was found in POD in $9(39.13 \%)$ cases. Uterine walls and uterine ligaments were found to be involved in 3 $(13.04 \%)$ and $2(8.70 \%)$ patients respectively (Table 4$)$.

On chromopertubation bilateral fallopian tubes were found to be patent in 16 patients $(69.57 \%)$. Unilateral and 
bilateral tubal blockage was seen in $4(17.39 \%)$ and 3 $(13.04 \%)$ patients respectively (Table 5).

Table 5: Status of fallopian tube on chromopertubation.

\begin{tabular}{|lll|}
\hline Tubal blockage & $\begin{array}{l}\text { No. of } \\
\text { patients }\end{array}$ & Percentage \\
\hline Both tubes patent & 16 & $69.57 \%$ \\
\hline Unilateral tubal blockage & 4 & $17.39 \%$ \\
\hline Bilateral tubal blockage & 3 & $13.04 \%$ \\
\hline Total & 23 & $100 \%$ \\
\hline
\end{tabular}

During laparoscopic procedures patients were either subjected to cystectomy $(30.43 \%)$, endometrioma drainage and fulguration (13.04\%) or adhesiolysis $(34.78 \%)$. In cases where deep seated endometriosis was found deep cauterization $(21.74 \%)$ was done (Figure 3 ).

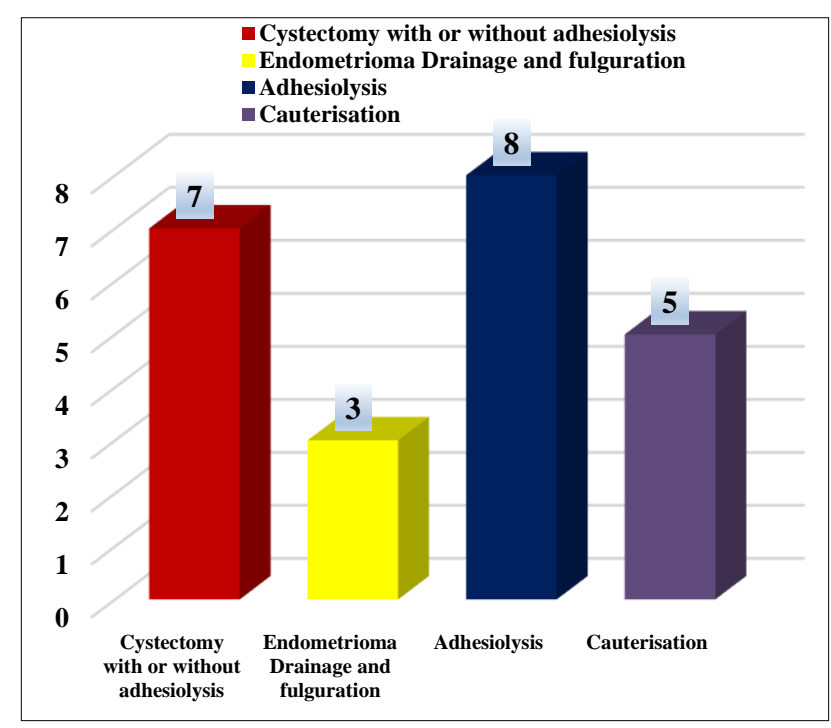

Figure 3: Surgical procedure done by laparoscopy.

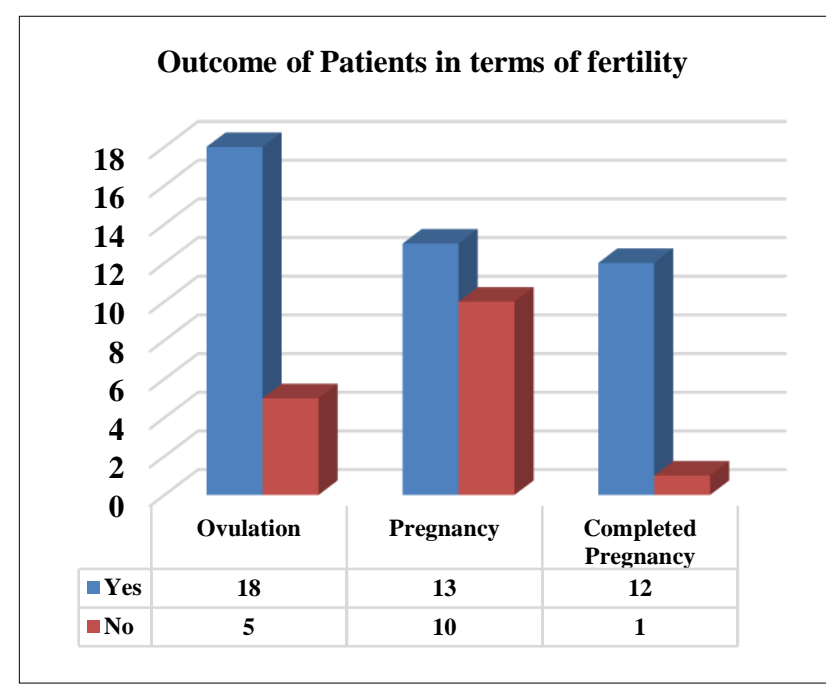

Figure 4: Outcome of patients in terms of successful pregnancy following laparoscopic procedure.
The patients were followed up for fertility outcome. Out of 23 patients who had undergone laparoscopic intervention ovulation occurred either with ovulation induction or spontaneously in $18(78.26 \%)$ patients. Out of these 18 patients intrauterine inseminisation was done in 10 patients out of which 7 patients conceived. 5 patients underwent IVF out of which 3 conceived whereas 3 patients conceived spontaneously. Pregnancy occurred within 1 year follow up period in 13 patients. Out of these 13 patients in whom pregnancy occurred 1st trimester spontaneous abortion occurred in 1 patient whereas 12 patients successfully completed their pregnancies. This study found that out of 18 patients who had successful ovulation following laparoscopic intervention $12(66.66 \%)$ patients completed their pregnancies successfully (Figure 4).

\section{DISCUSSION}

Endometriosis is one of the common causes of infertility and subfertility in young women. In this study majority of the patients $(47.83 \%)$ belonged to the age group of 25-30 years of the age. Many of the studies have reported similar age at which women are affected by endometriosis. Moradi $\mathrm{M}$ et al, conducted a qualitative descriptive study using semi-structured focus group discussions with 35 Australian women with endometriosis. ${ }^{9}$ In this study the mean age of the participants was found to be $31.1 \pm 10.4$ years (range 1753).

In this study the most common site was found to be ovaries which was found to be involved in all the cases. The other common sites involved were POD in 9 $(39.13 \%)$ cases. Uterine walls and uterine ligaments were found to be involved in $3(13.04 \%)$ and $2(8.70 \%)$ patients respectively. Valson $\mathrm{H}$ et al, conducted a prospective study of women with endometriosis. ${ }^{10}$ In this study a total of 200 patients who presented with symptoms of dysmenorrhea, dyspareunia, chronic pelvic pain with or/without infertility were studied. The diagnostic/operative laparoscopy was performed in 75 patients. Endometriosis was identified by laparoscopy in 50 cases and confirmed by histopathological examination. In this study also ovaries were one of the common sites for endometriosis account for almost $1 / 3^{\text {rd }}$ of the cases with endometriosis. Similar studies conducted by Macer ML et al, and Prescott $\mathrm{J}$ et al, also showed ovaries to be one of the common sites for endometriosis. ${ }^{11,12}$

Various studies have confirmed that the laparoscopic interventions are helpful in diagnosing as well as treating endometriosis related infertility. Various procedures which can be undertaken for treatment of endometriosis related infertility include cystectomy, endometrioma drainage and fulguration or adhesiolysis. In cases where deep seated endometriosis is found deep cauterization is usually undertaken. There are many studies which have concluded that laparoscopic surgeries are effective in 
treating mild and moderate endometriosis. These laparoscopic interventions are known to reduce overall pain and increases live birth and ongoing pregnancy rates. In this study out of 18 patients who had successful ovulation following laparoscopic intervention 12 patients completed their pregnancies successfully. Similar effectiveness of laparoscopic intervention is also reported by many other authors. Duffy JM et al, conducted a study to assess the effectiveness and safety of laparoscopic surgery in the treatment of painful symptoms and subfertility associated with endometriosis. ${ }^{13}$ For this purpose randomized controlled trials were selected in which the effectiveness and safety of laparoscopic surgery used to treat pain or subfertility associated with endometriosis was compared with any other laparoscopic or robotic intervention, holistic or medical treatment or diagnostic laparoscopy only. The study found that laparoscopic surgery was associated with an increased live birth or ongoing pregnancy rate and increased clinical pregnancy rate. Two studies collected data on adverse events (including infection, vascular and visceral injury and conversion to laparotomy) and reported no events in either arm. Other studies did not report this outcome. The authors concluded that there is moderate quality evidence that laparoscopic surgery to treat mild and moderate endometriosis reduces overall pain and increases live birth or ongoing pregnancy rates. Similar improvement in fertility after laparoscopic interventions was also reported by the authors such as Rizk B et al, and Lee $\mathrm{HJ}$ et al. ${ }^{14,15}$

\section{CONCLUSION}

Endometriosis is one of the common causes of infertility in young women. Though imaging can help in initial diagnosis, confirmation of endometriosis may require laparoscopy. Laparoscopy also have therapeutic role in endometriosis and have shown to be having encouraging results for treatment of infertility or subfertility in women.

Funding: No funding sources

Conflict of interest: None declared

Ethical approval: The study was approved by the Institutional Ethics Committee

\section{REFERENCES}

1. Parasar P, Ozcan P, Terry KL. Endometriosis: Epidemiology, Diagnosis and Clinical Management. Curr Obstet Gynecol Rep. 2017;6(1):34-41.

2. Alimi Y, Iwanaga J, Loukas M, Tubbs RS. The clinical anatomy of endometriosis: a review. Cureus. 2018;10(9):e3361.
3. Laghzaoui O, Laghzaoui M. Nasal endometriosis: apropos of 1 case. J Gynecol Obstet Biol Reprod (Paris). 2001;30(8):786-8.

4. Peterson CM, Johnstone EB, Hammoud AO. Risk factors associated with endometriosis: importance of study population for characterizing disease in the ENDO study. Am J Obstet Gynecol. 2013;208(6):451.e1-11.

5. Shah PR, Adlakha A. Laparoscopic management of moderate: severe endometriosis. J Minim Access Surg. 2014;10(1):27-33.

6. Adamson D. Surgical management of endometriosis. Semin Reprod Med. 2003;21(2):223-34.

7. Foti PV, Farina R, Palmucci S, Vizzini IA, Libertini $\mathrm{N}$, Coronella $\mathrm{M}$, et al. Endometriosis: clinical features, MR imaging findings and pathologic correlation. Insights Imag. 2018;9(2):149-72.

8. Mettler L, Schollmeyer T, Lehmann-Willenbrock E, Schüppler U, Schmutzler A, Shukla D, et al. Accuracy of laparoscopic diagnosis of endometriosis. JSLS. 2003;7(1):15-8.

9. Moradi M, Parker M, Sneddon A, Lopez V, Ellwood D. Impact of endometriosis on women's lives: a qualitative study. BMC Womens Health. 2014; $14: 123$.

10. Valson H, Kulkarni C, Teli B, Nazer T. Study of endometriosis in women of reproductive age, laparoscopic management and its outcome. Int J Reprod Contracept Obstet Gynecol. 2016;5:514-9.

11. Macer ML, Taylor HS. Endometriosis and infertility: a review of the pathogenesis and treatment of endometriosis-associated infertility. Obstet Gynecol Clin North Am. 2012;39(4):535-49.

12. Prescott J, Farland LV, Tobias DK, Gaskins AJ, Spiegelman D, Chavarro JE, et al. A prospective cohort study of endometriosis and subsequent risk of infertility. Hum Reprod. 2016;31(7):1475-82.

13. Duffy JM, Arambage K, Correa FJ, Olive D, Farquhar C, Garry R, et al. Laparoscopic surgery for endometriosis. Cochrane Database Syst Rev. 2014;(4):CD011031.

14. Rizk B, Fischer AS, Lotfy HA, Turki R, Zahed HA, Malik R, et al. Recurrence of endometriosis after hysterectomy. Facts Views Vis Obgyn. 2014;6(4):219-27.

15. Lee HJ, Lee JE, Ku SY, Kim SH, Kim JG, Moon $\mathrm{SY}$, et al. Natural conception rate following laparoscopic surgery in infertile women with endometriosis. Clin Exp Reprod Med. 2013;40(1):29-32.

Cite this article as: Sharfuddin S, Imam N, Pradhan A. Laparoscopic evaluation and prevalence of endometriosis among infertile women: a prospective study. Int J Reprod Contracept Obstet Gynecol 2020;9:1914-8. 